\title{
A phase-field approach to pneumatic fracture with anisotropic crack resistance
}

\author{
Carola Bilgen · Kerstin Weinberg
}

Received: 2 November 2020 / Accepted: 1 October 2021 / Published online: 24 October 2021

(C) The Author(s) 2021

\begin{abstract}
Phase-field models of fracture allow the prediction of crack propagation and crack patterns. In this contribution, externally driven fracture processes in linear and finite elasticity are investigated. Different approaches to consider pneumatic pressure and materials with non-isotropic crack resistance are studied, combined, and examined in detail. The versatility of the proposed models is proven by a series of numerical simulations in two and three dimensions.
\end{abstract}

Keywords Phase-field model · Fracture - Pneumatic fracture $\cdot$ Anisotropic crack resistance $\cdot$ Driven fracture

\section{Introduction}

The prediction of crack growth and fracture patterns is a central topic of fracture mechanics. For computation of such moving boundary problems, the phasefield approach to fracture has become popular recently, see Henry and Levine (2004), Karma et al. (2001), Bourdin et al. (2000), Bourdin et al. (2008), Miehe et al. (2010), Verhoosel and de Borst (2013), Borden et al. (2012), Miehe et al. (2015), Sargado et al. (2018)

\section{Bilgen \\ PMD Technologies AG, Martinshardt 19, 57074 Siegen, Germany}

K. Weinberg ( $\square)$

Lehrstuhl für Festkörpermechanik, Universität Siegen, 57076 Siegen, Germany

e-mail: kerstin.weinberg@uni-siegen.de among many others. Starting from the original phasefield fracture model of brittle elastic solids, the method has been extended to ductile fracture (Ambati and De Lorenzis 2016; Kuhn et al. 2016), to different coupled field problems like temperature or electric fields (Abdollahi and Arias 2012; Miehe et al. 2015; Zhou et al. 2018), to heterogenous solids (Teichtmeister et al. 2017; Bleyer and Alessi 2018; Li and Maurini 2019), to hydraulic fracture (Bourdin et al. 2012; Heider and Markert 2017; Wilson and Landis 2016; Zhou et al. 2018; Mikelić et al. 2019) and so on. In this respect, the present work contributes to the modeling of driven crack growth in solids with anisotropic behavior-a constellation which occurs in geological formations, in toughened glass or in strained biological tissues and often comes together with large deformations.

Various studies have been made on external load driven fracture, in particular, on hydraulic fracturing where a fluid under high pressure is injected into compressed soil to open cracks. Several phase-field approaches to hydraulic fracturing have been suggested, e.g. by Miehe et al. (2015, 2016), Mikelic et al. (2015, 2015) and others Luo and Ehlers (2015), Heider and Markert (2017) and Wilson and Landis (2016), usually assuming a homogeneous or porous bulk material. Pneumatic fracture can be interpreted as a simplification of hydraulic fracture in the sense that only the crack opening pressure is relevant and the coupling with fluid flow is negligible. Related loading regimes can be found in subsurface volcanic activities or pressurized vessel and comparable situations may also arise in the 
context of bio-materials. For example, a high blood pressure vasculature may lead to the rupture of vascular walls evoking major complications, cf. Yamashima and Friede (1984) and Ghilardi et al. (1993), Fig. 1.

By now most phase-field fracture simulations refer to homogeneous isotropic material. For the mentioned driven fracture situations this is not a realistic assumption. For instance, the typical shale formations of hydraulic fracturing have a heterogeneously layered structure. Such layers may have interfaces and directions with reduced crack resistance but also the stone itself may have inhomogeneities or be direction dependent. There are different ways to consider such fracture anisotropy. An explicit modeling of interfaces and its interaction with crack propagation is presented in Hansen-Dörr et al. (2017) and Bilgen et al. (2018) in the phase-field fracture context. Ghamgosar et. al. (2015) suggest to introduce various critical fracture energy densities $\mathcal{G}_{c}^{I}, \mathcal{G}_{c}^{I I}, \mathcal{G}_{c}^{I I I}$ for each rock direction. This technique was used in the early phase-field models of Adda-Bedia et al. (1999) to capture the preferred direction of crack propagation. Such adapted solution techniques facilitate the description of crack propagation in specific situations like pure mode I or mode II loading. In the last years it has been generalized to a Griffith energy tensor or, alternatively, a structural tensor included in the regularized variational phase-field formulation, cf. Clayton and Knap (2015), Teichtmeister et al. (2017), Liu and Juhre (2018) and Li et al. (2015). Such a tensor weights the partial derivatives in different directions separately.

In this work we present a model for pneumatic fracture in solids with non-isotropic fracture resistance. The model is novel, allows for linearized and finite deformations, and is based on a strict variational derivation. The direction dependent fracture resistance is mapped by a stochastic and by a deterministic approach. The paper is structured as follows: In Sect. 2 the phase-field fracture approach is introduced shortly. The equations of pneumatic fracture are specified in Sect. 3 where the crack opening pressure is incorporated as a moving boundary condition. Next, in Sect. 4 the non-isotropic fracture resistance is realized by a random field, a nonisotropic crack density function and a combination of both. All models are evaluated by series of numerical examples. Numerical simulations for pressure induced crack growth in materials with layer-wise fracture resistance are presented in Sect. 5. A short summary in Sect. 6 concludes the paper.

\section{The phase-field approach to fracture}

In phase-field fracture the material state is characterized by a continuous field $z(\boldsymbol{x}, t) \in[0,1]$ - the phasefield. The limit $z=1$ indicates the crack and $z=0$ marks the initial, unbroken state.

In the following we consider a solid with domain $\Omega \subset \mathbb{R}^{3}$ and boundary $\partial \Omega=\Gamma \subset \mathbb{R}^{2}$ deforming in a time interval $t \in\left[0, t_{\text {tot }}\right] \subset \mathbb{R}^{+}$. Its total potential energy shall be given by an elastic bulk energy with a Helmholtz free energy density $\Psi^{\mathrm{e}}$ and additional surface contributions due to cracking:

$\mathcal{E}=\int_{\Omega \backslash \Gamma} \Psi^{\mathrm{e}} \mathrm{d} \Omega+\int_{\Gamma(t)} \mathcal{G}_{c} \mathrm{~d} \Gamma \rightarrow$ optimum.

The surface integral in (1) results from the fact that crack growth involves the creation of new surfaces $\Gamma(t)$; the specific energy $\mathcal{G}_{c}$ corresponds to Griffith's critical energy release rate in brittle fracture. In phasefield fracture the sharp crack surface is approximated by a crack density function $\gamma=\gamma(z, \nabla z)$. In the present work we apply the second-order function

$\gamma(z, \nabla z)=\frac{1}{2 l_{c}} z^{2}+\frac{l_{c}}{2}|\nabla z|^{2}$,

where the length parameter $l_{c}$ weights the influence of the gradient term and determines the width of the diffuse zone. This form of the crack density function has commonly been used in the past years, cf. Bourdin et al. (2008), Miehe et al. (2010), Kuhn and Müller (2010) and Borden et al. (2012). The regularized total potential energy

$\mathcal{E}=\int_{\Omega}\left(\Psi^{e}+\frac{\mathcal{G}_{c}}{2 l_{c}}\left(z^{2}+l_{c}{ }^{2}|\nabla z|^{2}\right)\right) \mathrm{d} \Omega$

now has the form of an Ambrosio-Tortorelli functional Ambrosio and Tortorelli (1990) and states a non-local fracture theory here.

The phase field $z$ follows from potential energy optimization but is commonly subjected to a viscous regularization. We write here in general form

$\dot{z}(\boldsymbol{x}, z)=M Y(\boldsymbol{x}, z)$

with a mobility parameter $M\left[\mathrm{~m}^{2} / \mathrm{Ns}\right]$ and a local force symbolized by $Y$. The latter is usually the variation of 


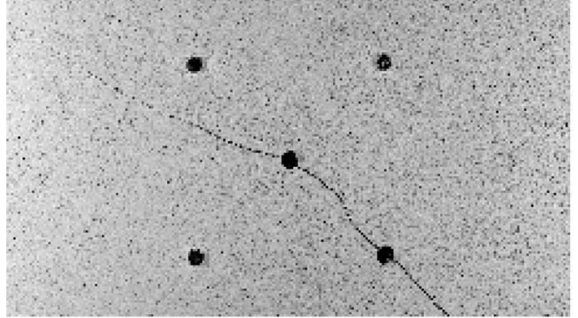

Fig. 1 Illustration of pressure driven fracture: a specimen cracks after pressing water in 3 of its holes (AlTammar et al. 2018) (left) and a vein rupturing by high blood

the energy density of (3) with respect to $z$ and can be decomposed into a crack driving force $Y^{e}$ and a crack resistance $Y^{f}$ such that $Y=Y^{e}-Y^{f}$, with crack resistance $Y^{f}=\delta_{z} \mathcal{G}_{c} \gamma(z)$.

There are different ways to formulate the crack driving force $Y^{e}$. In most theoretical works it is simply $Y^{e}=\delta_{z} \Psi^{e}$. Practical prerequisite, though, is a decomposition of the energy density which takes the asymmetry of fracture into account, i.e. only tensile states lead to crack propagation, $Y^{e}=\delta_{z} \Psi^{e+}$. However, there is no unique way to formulate such a decomposition and other physically motivated crack driving forces may be applied; we refer to Bilgen and Weinberg (2019) for a discussion. As an example, we consider here the local tensile stress state (Rankine stress) to be responsible for crack growth, i. e. the crack driving force is active when the maximum principal stress $\sigma_{I}=\max \left(\sigma_{a}\right), a \in\{1,2,3\}$ exceeds a critical value $\sigma_{c}$ :

$Y^{e}=\left\langle\sigma_{I}-\sigma_{c}\right\rangle_{ \pm}$

\subsection{Finite elasticity}

The phase-field model is adapted to finite deformations with a deformation mapping $\chi(\boldsymbol{X}, t): \Omega \times\left[0, t_{\text {tot }}\right] \rightarrow$ $\mathbb{R}^{3}$ and the deformation gradient

$\boldsymbol{F}=\nabla_{\boldsymbol{X}} \chi=\frac{\partial \chi}{\partial \boldsymbol{X}}$

where the quantities in capitals refer to the initial configuration. The determinant $J=\operatorname{det} \boldsymbol{F}$ represents the

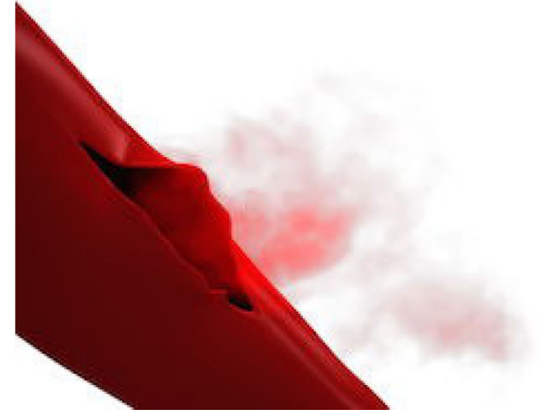

pressure (right), from https://www.healthtestingcenters.com/ what-you-need-know-about-cholesterol-and-cardiac-disease/

mapping of volume elements, $J=\mathrm{d} v / \mathrm{d} V$. The elastic strain energy density is defined as isotropic tensor function of $\boldsymbol{F}$ and can be formulated with its invariants. Specifically, we set here

$\Psi^{e}=\Psi^{e}\left(\bar{I}_{1}, \bar{I}_{2}, J\right)$

with modified invariants based on the isochoric split of the deformation gradient, $\boldsymbol{F}=J^{1 / 3} \overline{\boldsymbol{F}}$.

$\bar{I}_{1}=\operatorname{tr}\left(\overline{\boldsymbol{F}}^{T} \overline{\boldsymbol{F}}\right)=J^{-2 / 3} \boldsymbol{F}: \boldsymbol{F}$
$\bar{I}_{2}=\operatorname{tr}\left(\operatorname{cof}\left(\overline{\boldsymbol{F}}^{T} \overline{\boldsymbol{F}}\right)\right)=J^{4 / 3} \boldsymbol{F}^{-T}: \boldsymbol{F}^{-T}$

For later reference, we also define the right CauchyGreen deformation tensor $\boldsymbol{C}=\boldsymbol{F}^{T} \boldsymbol{F}$ and the cofactor tensor $\boldsymbol{H}=\operatorname{cof}(\boldsymbol{F})$ which represents the mapping of surface elements in a deformation. With the latter, the second invariant can be formulated as

$\bar{I}_{2}=J^{4 / 3} \operatorname{tr}(\operatorname{cof} \boldsymbol{C})=J^{4 / 3} \boldsymbol{H}: \boldsymbol{H}$.

As proposed in Hesch et al. (2017) and analyzed in Thomas et al. (2020) we consider the tensioncompression asymmetry of fracture by a decomposition of the invariants into tensile $(+)$ and compressive (-) components

$$
\begin{gathered}
\bar{I}_{1}^{ \pm}=3+\left\langle\bar{I}_{1}-3\right\rangle^{ \pm}, \quad \bar{I}_{2}^{ \pm}=3+\left\langle\bar{I}_{2}-3\right\rangle^{ \pm}, \\
J^{ \pm}=1+\langle J-1\rangle^{ \pm} .
\end{gathered}
$$


This allows to formulate the elastic strain energy density in a decomposed way,

$\Psi^{e}=g(z) \Psi^{e+}+\Psi^{e-}$.

The degradation function $g(z)$ is introduced to account for the material loss; we set here $g(z)=(1-z)^{2}$. In the following we apply a Neohookean material model with strain energy density

$$
\begin{aligned}
\Psi^{e}= & g(z)\left(\frac{\mu}{2}\left(\bar{I}_{1}^{+}-3\right)+\frac{K}{2}\left(J^{+}-1\right)^{2}\right) \\
& +\frac{\mu}{2}\left(\bar{I}_{1}^{-}-3\right)+\frac{K}{2}\left(J^{-}-1\right)^{2}
\end{aligned}
$$

where $K$ is the bulk modulus and $\mu$ the initial shear modulus. Naturally, the simple volumetric term in (12) can easily be replaced by a convex expression, which then renders the energy density to be polyconvex. For detailed information we refer to Hesch et al. (2017) and Weinberg and Hesch (2015).

\subsection{Linear elasticity}

The equations can equivalently be formulated for linear elasticity with strains $\epsilon(\boldsymbol{u})=\frac{1}{2}\left(\nabla \boldsymbol{u}+\nabla^{T} \boldsymbol{u}\right)$ of a displacement field $\boldsymbol{u}(\boldsymbol{x})$. The invariants (8) reduce to $\bar{I}_{2}=0$ and

$$
\begin{gathered}
\bar{I}_{1}=2 \operatorname{tr}\left(\epsilon^{2}\right)+3 \\
J=1+\operatorname{tr}(\epsilon) .
\end{gathered}
$$

The elastic strain energy density $\Psi^{e}=\Psi^{\mathrm{vol}}+\Psi^{\mathrm{dev}}$ has a volumetric part $\Psi^{\mathrm{vol}}=\frac{1}{2} K(\operatorname{tr} \epsilon)^{2}$ and an incompressible part $\Psi^{\text {dev }}=2 \mu \operatorname{tr}\left(\epsilon^{2}\right)$.

The decomposition of the invariants into tensile and compressive components gives

$\bar{I}_{1}^{ \pm}=3+\left\langle 2 \operatorname{tr} \epsilon^{2}\right\rangle^{ \pm}, \quad J^{ \pm}=1+\langle\operatorname{tr} \epsilon\rangle^{ \pm}$.

Then, the volumetric part of the energy $\Psi^{\mathrm{vol}}=$ $\frac{1}{2} K(J-1)^{2}$ corresponds to $\Psi^{\mathrm{vol}}=\frac{1}{2} K(\operatorname{tr} \epsilon)^{2}$ in linear elasticity. Accordingly, the decomposition of the energy conforms with the split of the linear elastic energy proposed by Amor et al. (2009) and we conclude that the proposed decomposition of the strain energy density (11) can be adapted to small deformations as well.
For later reference we state here another common energy split, namely the $\lambda-\mu$ split, with Lámeconstants $\lambda=K-\frac{2}{3} \mu$ and $\mu$,

$$
\begin{aligned}
\Psi^{e}= & g(z)\left(\frac{1}{2} \lambda\langle\operatorname{tr} \epsilon\rangle^{+2}+\mu \epsilon^{+}: \epsilon^{+}\right)+\frac{1}{2} \lambda\langle\operatorname{tr} \epsilon\rangle^{-2} \\
& +\mu \epsilon^{-}: \epsilon^{-}
\end{aligned}
$$

\section{Pneumatic fracture}

In this section we consider pneumatic fracture, i.e., crack growth in a compressed medium driven by a pressurized state within the crack. The applied pressure is modeled as a moving boundary condition. The model is similar to the approach of Bourdin et al. (2012), who considered small deformations only, and derived here in a variational form for small and finite deformations.

\subsection{Pressure driven crack growth}

We start with the potential energy (1) of the body in its regularized form (3) and focus on the strain energy density (12), its small strain counterpart or the corresponding formulation (16). The pressurized gas injected for pneumatic cracking is associated with another energy contribution and so we add a pneumatic term

$\mathcal{E}=\int_{\Omega}\left(\Psi^{e}+\Psi^{\text {pneu }}+\frac{\mathcal{G}_{c}}{2 l_{c}}\left(z^{2}+l_{c}^{2}|\nabla z|^{2}\right)\right) \mathrm{d} \Omega$

which has the form

$\Psi^{\text {pneu }}=-\bar{p}(z ; t) \operatorname{det} \boldsymbol{F}$

or, for linearized strains,

$\Psi^{\text {pneu }}=-\bar{p}(z ; t) \operatorname{tr} \epsilon$.

Hereby $\bar{p}(z ; t)$ is the applied pressure. This pressure field is coupled with the phase field $z$ and may vary in time. Within a crack at position $\boldsymbol{x}$ it is modeled as:

$\bar{p}(z(\boldsymbol{x}, t) ; t)=\left(p_{0}(\boldsymbol{x})+p_{1}(\boldsymbol{x}, t)\right) z(\boldsymbol{x}, t)$

The pressure is active when the phase-field is $\neq 0$ and acts on the crack then. At the crack flanks the static 
boundary condition $\boldsymbol{\sigma} \cdot \boldsymbol{n}=0$ typically holds. For a crack with pressure $\bar{p}$ it changes to $\boldsymbol{\sigma} \cdot \boldsymbol{n}=\bar{p}$ or $\boldsymbol{\sigma}$. $\boldsymbol{n}-\bar{p}=0$. However, in the diffuse crack zone of the phase-field model the normal to a crack flank $\boldsymbol{n}$ cannot be computed directly and so the additional pressure component has to be added in the variational approach. The specific pressure field (20) is a matter of modeling; the linear form corresponds to the model of Bourdin (2012) for small strains, see Remark 3.6.

The first Piola-Kirchhoff stress tensor is derived from the strain energy density as

$\boldsymbol{P}=\frac{\partial \Psi}{\partial \boldsymbol{F}}$

and relates to the Cauchy stress tensor $\sigma$ by $\sigma=$ $J^{-1} \boldsymbol{P} \boldsymbol{F}^{T}$, cf. Holzapfel (2006). With (17) and (18) it follows

$\boldsymbol{P}(\boldsymbol{F}, z ; t)=\frac{\partial \Psi^{e}}{\partial \boldsymbol{F}}+\frac{\partial \Psi^{\text {pneu }}}{\partial \boldsymbol{F}}=\boldsymbol{P}^{e}(\boldsymbol{F}, z)-\bar{p}(z, t) \boldsymbol{H}$,

where we denote the Neohookean part of the stresses by $\boldsymbol{P}^{e}$ and $\boldsymbol{H}=\operatorname{cof}(\boldsymbol{F})$ as above.

Alternatively we derive the linear-elastic stresses as the work conjugate to the linearized strains,

$\boldsymbol{\sigma}(\epsilon, z)=\frac{\partial \Psi^{e}}{\partial \epsilon}+\frac{\partial \Psi^{\text {pneu }}}{\partial \epsilon}$

which gives with $\sigma^{e}=\partial \Psi^{e} / \partial \epsilon$ the modified stresses

$\boldsymbol{\sigma}(\epsilon, z ; t)=\boldsymbol{\sigma}^{e}(\epsilon, z)-\bar{p}(z ; t) \mathbf{1}$.

Please note that this is a (diffuse) moving boundary condition since the loaded boundary grows with crack propagation.

\subsection{Proof of concept}

To demonstrate applicability we study a two dimensional notched plate of size $100 \times 100 \mathrm{~mm}^{2}$. The mesh consists of $256 \times 256$ quadratic B-spline finite elements. Here we use the spline functions for convenience only; they provide here a similar approximation as classical Lagrangian elements of quadratic order. While the boundaries are constrained in all directions, a notch
Table 1 Material parameters of the numerical simulations

\begin{tabular}{lll}
\hline Parameter & Value & Unit \\
\hline$E$ & 50,000 & {$\left[\mathrm{~N} / \mathrm{mm}^{2}\right]$} \\
$v$ & 0.2 & {$[-]$} \\
$\mathcal{G}_{c}$ & $75 \times 10^{-3}$ & {$[\mathrm{~N} / \mathrm{mm}]$} \\
$\sigma_{c}$ & 31.6228 & {$\left[\mathrm{~N} / \mathrm{mm}^{2}\right]$} \\
\hline
\end{tabular}

of length $16 \mathrm{~mm}$ in the middle of the domain is prescribed by fixing the phase-field parameter to $z=1$, see left plot of Fig. 2. The numerical simulation uses the Neohookean model with the decomposed invariants (10). The material parameters correspond to concrete and are given in Table 1; the mobility of Eq. (4) is $M=1 \mathrm{~mm}^{2} / \mathrm{Ns}$ whereby the time is normalized, $t=t_{n} \cdot p \Delta t, \Delta t=1 \mathrm{~s}$. The nonlocal length parameter is $l_{c}=4.5 \cdot 10^{-4} \mathrm{~mm}$. For the applied pressure the values $p_{0}=10 \mathrm{~N} / \mathrm{mm}^{2}$ and $p_{1}=10^{4} \cdot t_{n} \mathrm{~N} / \mathrm{mm}^{2}$ are chosen, with $t_{n} \in\{1, \ldots, 75\}$.

The growing crack is shown in Fig. 2 at different (quasi)time steps $t_{n}$. The crack propagates horizontally until it branches in the vicinity of the boundaries. The position of branching depends on the amount of applied pressure. For the values chosen here the results correspond well to other models in literature, cf. Bourdin et al. (2012), Mikelic et al. (2015) and Miehe and Mauthe (2016).

We remark that the irreversibility of crack growth in (17) is enforced through an inequality constraint $\dot{s} \leq$ 0. In Wheeler et al. (2014) an augmented Lagrangian algorithm for energy optimization is proposed instead.

\subsection{Validation}

In order to validate our solution we make use of analytical expressions for the two-dimensional Griffith crack, which go back to Sneddon \& Lowengrub (1969), Sects. 2.1 and 2.4. The critical energy release rate of a pressurized crack of length $2 a$ is $\mathcal{G}_{c}=\pi p^{2} a / E^{\prime}$ with the elastic parameter $E^{\prime}=E /\left(1-v^{2}\right)$. Consequently, the critical pressure of crack opening follows as

$p_{c}=\sqrt{\frac{G_{c} E^{\prime}}{\pi a}}$.

Accordingly, the crack opening displacement can be integrated to give the $2 \mathrm{D}$ volume of the crack, $V=$ 

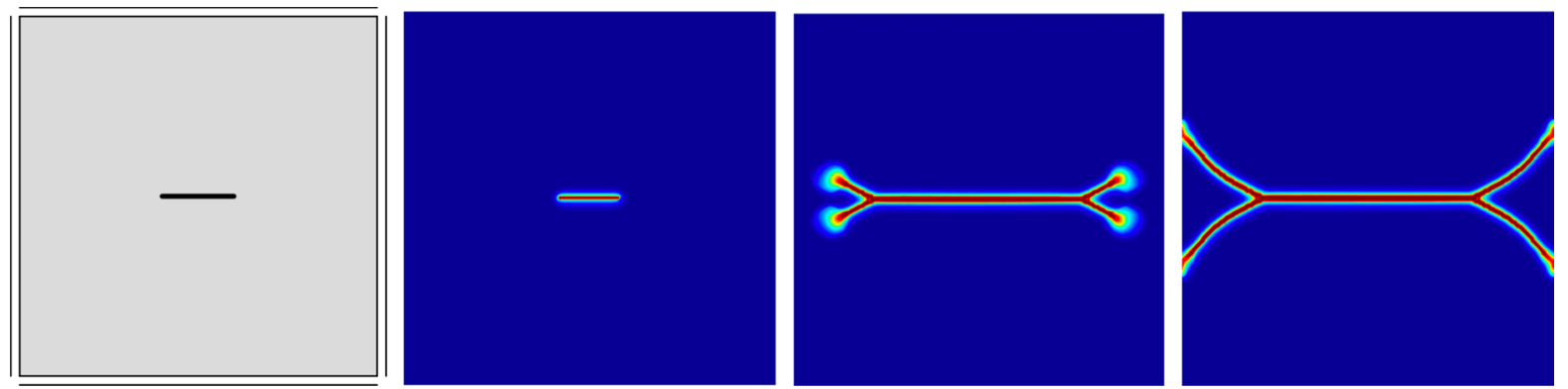

Fig. 2 Boundary conditions (left) and crack propagation of the pressure driven fracture at different time steps $t_{n} \in\{1,60,74\}$

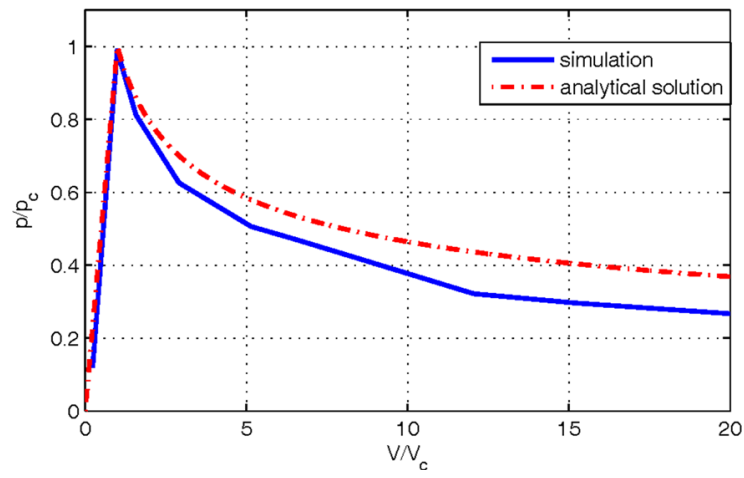

Fig. 3 Comparison of the proposed model with the analytical pressure-volume relation (27)

$2 \pi p a^{2} / E^{\prime}$, which has a critical value at pressure $p_{c}$, i.e.

$V_{c}=\sqrt{\frac{4 \pi \mathcal{G}_{c} a^{3}}{E^{\prime}}}$.

Injection of a volume less then $V_{c}$ gives no crack growth but a linear pressure increase in the elastically deforming body. After $V_{c}$ is reached, the crack starts propagating. Eliminating $a$ from $p_{c}$ and $V_{c}$, we obtain the corresponding pressure-volume relation in closed form:

$p(V)=\sqrt[3]{\frac{2 E^{\prime} \mathcal{G}_{c}^{2}}{\pi V}}$.

In Fig. 3 the result of our numerical simulation is compared with the analytical solution (27). Hereby the pressure is prescribed and the resulting $2 \mathrm{D}$ volume is calculated from the displacement of the crack flanks. We see the linear pressure increase in the body until the critical pressure is reached; then the crack grows and the pressure decays. Both curves have the same shape but the pressure during crack growth is smaller in our simulation than in the analytical solution. We remark that we prescribe the crack pressure but do not control the volume. Here and below, we define a crack boundary at phase-field values of $z \geq 0.9$; other values may result in different volumes. The same holds for further phasefield model details like the degeneration function $g(z)$ and length parameter $l_{c}$. For related studies we refer to Bourdin et al. (2012) and Mikelic et al. (2015).

Additionally we compare the crack opening displacements. According to Sneddon \& Mott (1946), the opening of a fully pressurized crack follows the equation

$w=c p \sqrt{1-\frac{r^{2}}{a^{2}}}$

with $c=2 a / E^{\prime}$ and radius $r \equiv x$ for the twodimensional model, and with $c=4 a /\left(\pi E^{\prime}\right)$ for a threedimensional penny-shaped crack.

In the left plot of Fig. 4, analytical and numerical results for the two-dimensional model are plotted. Shown are the displacements at two points, namely at position $x_{1}=0.4 a$ and $x_{2}=0.8 a$. The analytical solution is determined according to Eq. (28) for each time step with the respective applied pressure $p(t)$ for values $p_{0}, p_{1}$ as above and time step $\Delta t=0.01 \mathrm{~s}$. The simulations show a good agreement with the analytical calculations for the first stages (in the elastic range) but crack growth changes the overall situation. The analytical solution considers the displacements at a given (initial) crack length, whereas the computations now refer to larger cracks and so a relaxation and an overall smaller displacement follows. To illustrate the situation 
we plotted the displacement at position $x=1.2 a$, i.e., on a point which only opens during crack growth.

In the right plot of Fig. 4, the corresponding results of a three-dimensional computation are displayed, see Sect. 5.2 for the details. Here the displacements are measured at material points with local radius $r_{1}=0.4 a$ and $r_{2}=0.8 a$. In both cases, we see again that the crack expansion is smaller than the analytical solution suggests, and due to the linear pressure increase, the crack width also increases linearly. The computation is stopped here after the crack has reached approximately 1.5 of the initial crack length $a$ because the configuration then deviates from the assumptions of the analytical solution considerably.

\subsection{Two notch crack propagation}

In another two-dimensional simulation we keep mesh, boundary conditions, material and pressure values as above but adapt the phase field in such a way that now two notches are prescribed and loaded with the applied pressure. The resulting phase-field snapshots are presented in Fig. 5. It can be seen that the cracks grow towards the boundaries until the horizontal notch reaches the vertical one. A corresponding crack path can be found in Miehe and Mauthe (2016).

\subsection{Crack propagation with a Rankine stress model}

To illustrate the effect of the alternative crack driving force (5) in the context of pressure driven crack growth, the simulation of Sect. 3.2 is repeated. The setting and also the pressure remain the same and the related phasefield snapshots are shown in Fig. 6. Compared to the results from the energetic crack driving force, it can be seen that the crack branches later such that the boundaries influence the crack tip in their vicinity only. This effect stems from the fact that only tensile stress states contribute to crack growth here whereby in the energy driven model also shearing has a certain contribution, cf. Bilgen and Weinberg (2019).

\subsection{Remarks}

In our approach the applied pressure results in an additional energy contribution. Bourdin et al. (2012) presented a similar way to incorporate the pressure as an additional crack driving force, preserving $\Gamma$ convergence of the regularized formulation. They extended the total potential energy by the work of a pressure force $\tilde{p}$ such that:

$\mathcal{E}=\int_{\Omega_{0}}\left(\Psi^{e}+\mathcal{G}_{c} \gamma+\tilde{p} \boldsymbol{u} \cdot \nabla z\right) \mathrm{d} V$

For small strains and one pressurized crack this formulation is basically identical to ours in Eq. (17). To show this we set $p_{0}=0$ and $p_{1}=\tilde{p}$ in Eq. (19), and separate the pneumatic energy contribution of (17) to state

$$
\begin{aligned}
& \int_{\Omega_{0}} \Psi^{\text {pneu }} \mathrm{d} \Omega=\int_{\Omega_{0}}-\tilde{p} \operatorname{tr} \epsilon \cdot z \mathrm{~d} \Omega \\
& =-\int_{\Omega_{0}} \tilde{p} \nabla \boldsymbol{u}: \mathbf{1} z \mathrm{~d} \Omega=\int_{\Omega_{0}} \tilde{p} \boldsymbol{u} \cdot \nabla z \mathrm{~d} \Omega
\end{aligned}
$$

whereby the last step presumes neglected boundary effects. Then the result equals the pressure related term of Bourdin et al. (2012), eq. (29). Clearly, the model of Bourdin et al. (2012) is restricted to linear elasticity whereas we state it in a general form here.

Additionally we remark that the proposed type of pressure boundary condition can be used in the same way for two and three dimensional simulations, cf. Sect. 5.2.

\section{Phase-field model with non-isotropic crack resistance}

Geological and also organic materials are commonly not smoothly homogenous but have inclusions, softer and harder layers, and different strength in different directions. That influences also their resistance to fracture and therefore we will consider such properties in the following. We remark that we do not aim at modeling the material's anisotropy because there exist established strategies for linear and finite elasticity Marsden et al. (1994) and Holzapfel (2006). Here we focus on non-isotropic crack resistance.

\subsection{Random variations in tensile resistance}

At first we model variations of the crack resistance due to inhomogeneities in the solid. This can be done by 

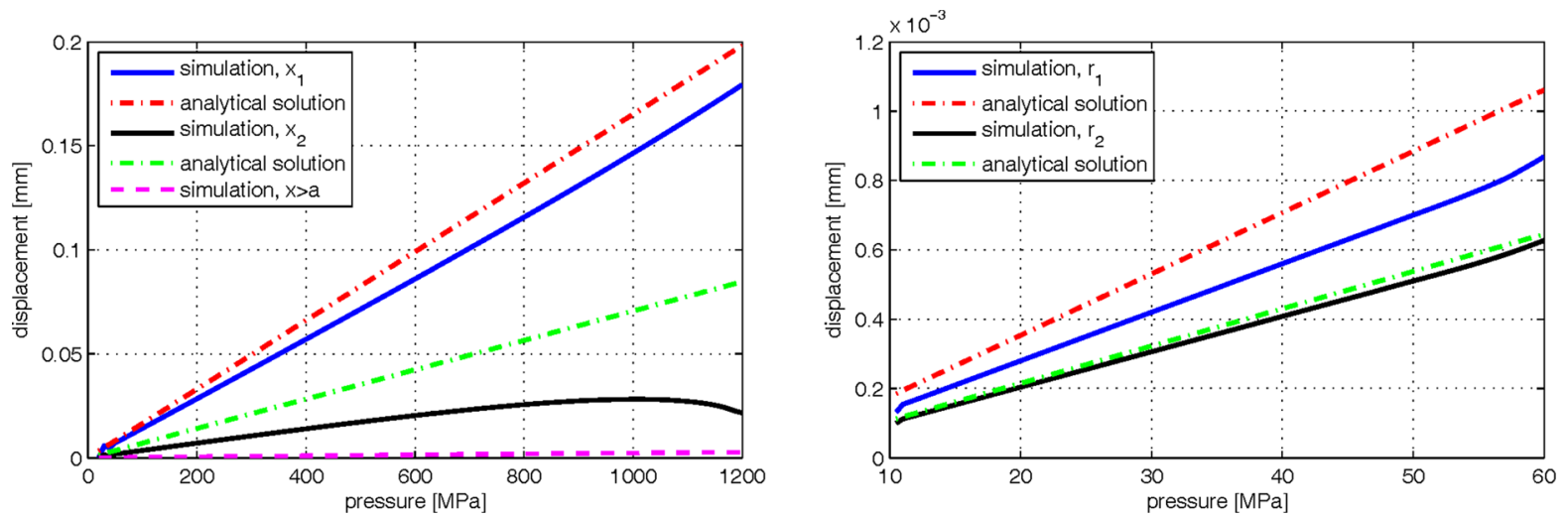

Fig. 4 Crack opening displacements at $x_{1}=0.4 a, x_{2}=0.8 a$ in the two-dimensional model (left) and at $r_{1}=0.4 a, r_{2}=0.8 a$ in the three-dimensional model of Sect. 5.2 with a penny-shaped crack (right)
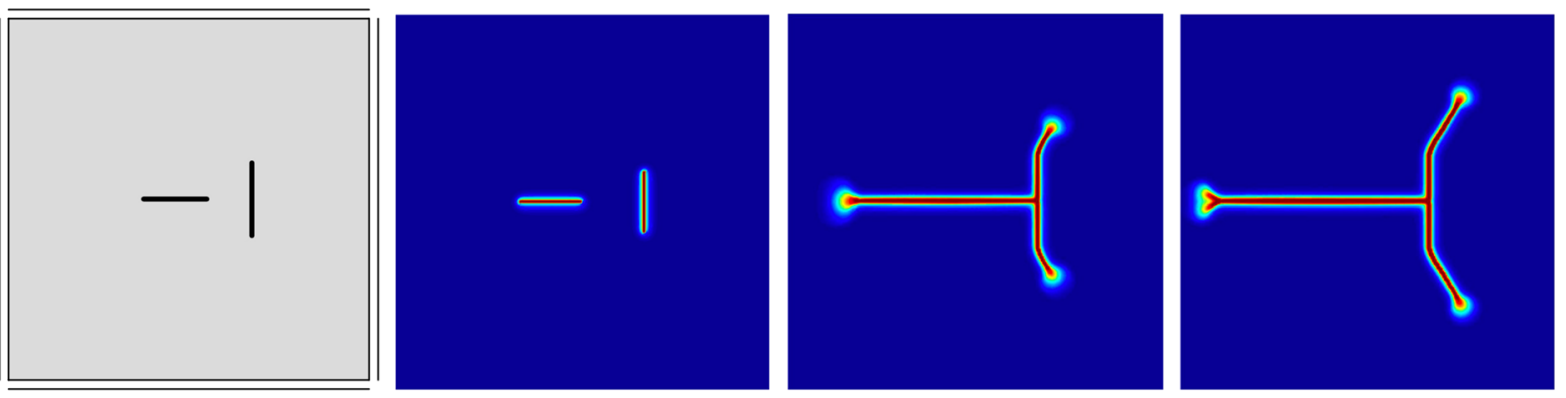

Fig. 5 Boundary conditions (left) and crack propagation of the pressure driven fracture at different time steps $t_{n} \in\{1,45,60\}$
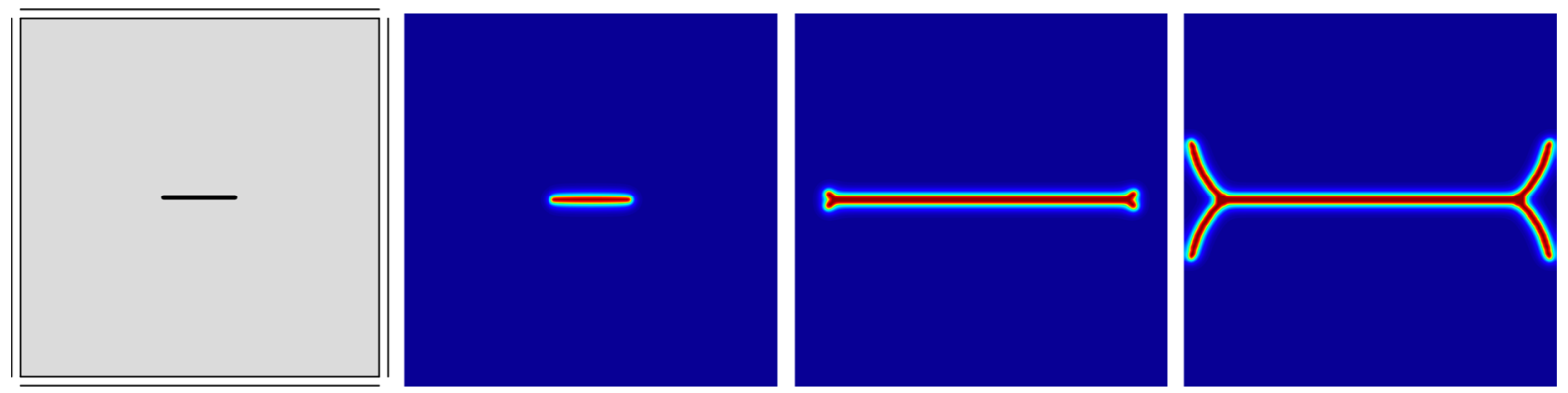

Fig. 6 Boundary conditions (left) and crack propagation of the pressure driven fracture at different time steps $t_{n} \in\{1,60,74\}$

explicitly accounting for two or more phases of material, cf. Hansen-Dörr et al. (2017), or using a stochastically 'smeared' approach. Here, the basic idea is to vary the critical parameters of the phase-field model, in particular the critical energy release rate $\mathcal{G}_{c}$ and/or the critical stress $\sigma_{c}$, by means of a superposed function $\xi(\boldsymbol{x})$ that influences the crack resistance $Y^{f}$ of Eq. (4). The total potential energy (3) then has the form:
$\mathcal{E}=\int_{\Omega_{0}} \Psi^{e}+\xi(\boldsymbol{x}) \mathcal{G}_{c} \gamma(z) \mathrm{d} V$

One way to incorporate local variation is to model $\xi(\boldsymbol{x})$ stochastically distributed over the whole domain. To do so, we apply an operator scaling stable random field (OSSRF). Such a OSSRF maps the deviations of the material from its ideal state and has been used for phase-field modeling before, cf. Anders et al. 
(2011). The OSSRF is based on time and space continuous Lévy walks which are, figuratively speaking, a mix of long trajectories and short random movements. A stable Lévy motion is a self-similar process with a scaling parameter $H>0$, the Hurst index. Operatorscaling self-similar processes allow the Hurst index to vary with the coordinates. Thus we choose for $(\boldsymbol{x}, t) \in$ $\Omega \times\left[0, t_{\text {tot }}\right]$ and parameters $0<H_{1}, H_{2}, H_{3}<1$ a random field of the form

$$
\begin{gathered}
\xi(\boldsymbol{x}, t)=\operatorname{Re} \int_{\Omega \times\left[0, t_{\mathrm{tot}}\right]}\left(e^{i \boldsymbol{x} \cdot \boldsymbol{y}}-1\right)\left(e^{i\left(t_{\max }-t\right) s}-1\right) \\
\cdot \psi_{1}(\boldsymbol{y}) \cdot \psi_{2}(s) P(\mathrm{~d} \boldsymbol{y} \times \mathrm{d} s)
\end{gathered}
$$

where

$$
\begin{aligned}
& \psi_{1}(\boldsymbol{y})=\left(\left|\boldsymbol{y} \cdot \boldsymbol{\theta}_{1}\right|^{2 H_{1}}+\left|\boldsymbol{y} \cdot \boldsymbol{\theta}_{2}\right|^{2 H_{2}}\right)^{-\frac{H_{1}+H_{2}}{4}-\frac{1}{2}}, \\
& \psi_{2}(s)=\frac{1}{|s|^{\frac{1+2 H_{3}}{2}}} .
\end{aligned}
$$

The variables $\boldsymbol{\theta}_{1}, \boldsymbol{\theta}_{2}$ are normalized eigenvectors of a scaling matrix with given eigenvalues $\mathrm{H}_{1}^{-1}, \mathrm{H}_{2}^{-1}$, respectively. The field (32) is anisotropic if the spatial scaling relation has different Hurst indices in the - not necessarily orthogonal - directions of the eigenvectors $\boldsymbol{\theta}_{1}, \boldsymbol{\theta}_{2}$. The measure $P(\mathrm{~d} \boldsymbol{y} \times \mathrm{d} s)$ indicates a complex-valued independently scattered isotropic random Gaussian measure. The Hurst index $\mathrm{H}_{3}$ refers to time; however, we set $\xi(\boldsymbol{x}, t)=\xi(\boldsymbol{x})$ and fix the temporal coordinate here. For mass conservation the condition

$$
\int_{\Omega} \xi(x, t) \mathrm{d} x=0
$$

has to be fulfilled. In Figure 7 the orientations and the random field are demonstrated exemplarily.

With this condition, the OSSRF does not affect the physics of the model, see Anders et al. (2011) for a discussion. In contrast to Gaussian white noise the OSSRF allows to model long range dependencies in space and can map anisotropic behavior.

\section{Proof of concept}

To demonstrate the influence of the $\operatorname{OSSRF} \xi(\boldsymbol{x})$ we perform a mode-I tension test. A square domain of size $100 \times 100 \mathrm{~mm}^{2}$ with a prescribed notch is loaded by tension, see left plot of Fig. 8. The mesh consists of $200 \times 200$ quadratic B-spline elements; the lengthscale parameter is $l_{c}=1 \mathrm{~mm}$. The inhomogeneity of the material with parameters of Table 1 is modeled with field (32) with $H_{1}=0.9, H_{2}=1$ and $t=t_{0}$, where $t_{0}$ is fixed. The results for two realizations are demonstrated in Fig. 8. In both cases the crack path is influenced by the random field. We remark that these investigations are only exemplarily. The OSSRF enables layer-like properties within a material, e.g. in shale formations or in plagued arteries. However, it may be difficult to adapt it to a given material behavior with preferred directions. Therefore, we suggest a deterministic tensor formulation in the following.

\subsection{Deterministic conductance tensor}

The elastic strain energy density (7) is per definition an isotropic tensor function whose arguments are based on the right Cauchy-Green deformation tensor $\boldsymbol{C}$. It is invariant against rigid body movements, i.e. it holds $\Psi^{e}(\boldsymbol{C})=\Psi^{e}\left(\boldsymbol{Q C} \boldsymbol{Q}^{T}\right)=\Psi^{e}\left(\boldsymbol{Q} \boldsymbol{F}^{T} \boldsymbol{F} \boldsymbol{Q}^{T}\right)$ for arbitrary rotations $Q \in \mathrm{SO}(3)$ Holzapfel (2006); $\mathrm{SO}$ (3) is the special orthogonal group of three dimensional rotations. The formulation in invariants (7) describes material with isotropic properties, i.e.

$\Psi^{e}(\boldsymbol{Q F})=\Psi^{e}(\boldsymbol{F})$

for all $Q \in \mathrm{SO}(3)$. For materials with a preferred direction, for instance as a consequence of fibers, the energy formulation needs to be modified. The simplest way to account for a reinforced direction $\boldsymbol{a}$ is to introduce an additional term, a structural tensor $\boldsymbol{a} \otimes \boldsymbol{a}$, in the energy form. One can easily verify that this also gives an isotropic tensor function, now in two arguments, $\Psi^{e}(\boldsymbol{C}, \boldsymbol{a} \otimes \boldsymbol{a})=\Psi^{e}\left(\boldsymbol{Q C} \boldsymbol{Q}^{T}, \boldsymbol{Q a} \otimes \boldsymbol{a} \boldsymbol{Q}^{T}\right)$. Additional directions may be considered by additional structural tensors.

We adapt this approach here and consider material with isotropic elastic properties but a direction dependent resistance to cracking. As above we assume a modified resistance in a direction $\boldsymbol{a}=\alpha \boldsymbol{a}_{0}$ whereby $\boldsymbol{a}_{0}$ is a normalized vector and $\alpha$ its relative length. Each direction defines a conductance tensor $\alpha^{2} \boldsymbol{a}_{0} \otimes \boldsymbol{a}_{0}$ which now enters the crack resistance part of energy density (3) as an additional term. Formulating the crack density function (2) for two (or more) preferred directions it reads 
Fig. 7 Random field $\xi$ with $\boldsymbol{\theta}_{1}=e^{i \cdot 0.3 \pi}, \boldsymbol{\theta}_{2}=e^{i \cdot 0.2 \pi}$ $H_{1}=H_{2}=0.5$; the colors describe the intensity/magnitude of $\xi$ (at a fixed time)
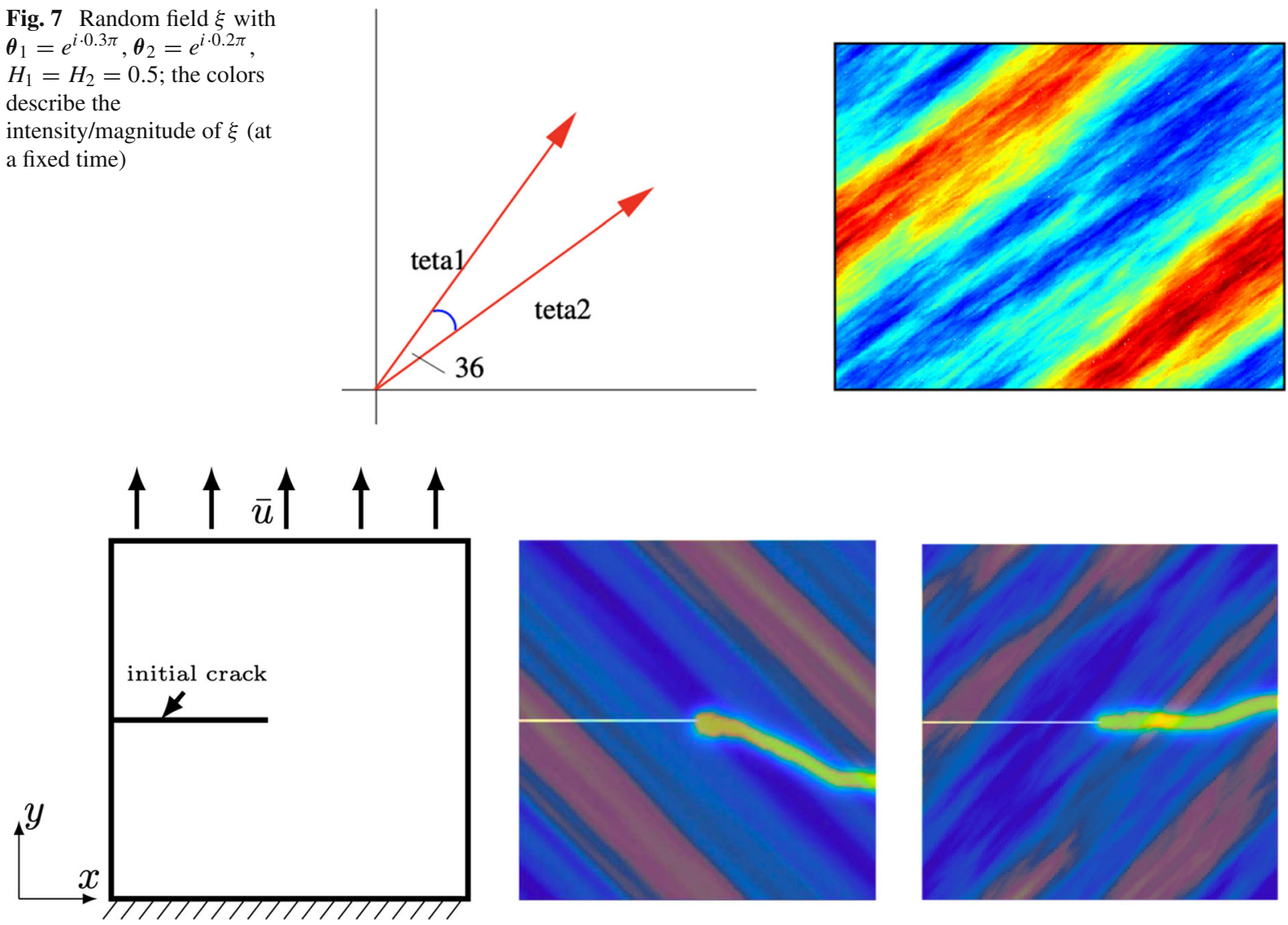

Fig. 8 Mode-I crack propagation with a crack resistance controlled the random field $\xi\left(\boldsymbol{x}, t_{0}\right)$

$$
\begin{aligned}
\gamma\left(z, \boldsymbol{a}_{1}, \boldsymbol{a}_{2}, \ldots\right)= & \frac{1}{2 l_{c}} z^{2} \\
& +\frac{l_{c}}{2} \nabla z \cdot \nabla z+\frac{l_{c}}{2} \alpha_{1}^{2} \nabla z \cdot \boldsymbol{a}_{1} \otimes \boldsymbol{a}_{1} \cdot \nabla z \\
& +\frac{l_{c}}{2} \alpha_{2}^{2} \nabla z \cdot \boldsymbol{a}_{2} \otimes \boldsymbol{a}_{2} \cdot \nabla z+\ldots
\end{aligned}
$$

Similar to (34) it holds for an isotropic crack density function the equation

$\gamma(z, Q \nabla z)=\gamma(z, \nabla z)$

for all $Q \in \mathrm{SO}(3)$; in the case of anisotropy the condition is valid only for all $Q \in \mathcal{B} \subset \mathrm{SO}$ (3) with an appropriate subset $\mathcal{B}$ of the orthogonal group defined by the conductance vectors $\boldsymbol{a}_{1}, \boldsymbol{a}_{2} \ldots$ of preferred crack directions.

There may be several directions of modified crack resistance which result in additional terms in (35) but in the following we focus on a unidirectional conductance for simplicity. Then, the crack density function can be written in the form

$\gamma\left(z, \boldsymbol{a}_{0}\right)=\frac{1}{2 l_{c}} z^{2}+\frac{l_{c}}{2} \nabla z \cdot\left(\mathbf{1}+\alpha^{2} \boldsymbol{a}_{0} \otimes \boldsymbol{a}_{0}\right) \cdot \nabla z$

where the relative vector length $\alpha$ plays the role of a weight, $\alpha^{2}$. Choosing $\alpha=0$ leads to isotropic material behavior as Eq. (37) reduces to Eq. (2). High values of $\alpha$ weight the conductance tensor and pronounce the preferred crack direction; a negative sign $-\alpha^{2}$ would result in a higher crack resistance of that direction.

\subsubsection{Mode-I tension test with different weights}

To illustrate the influence of the weight $\alpha^{2}$ in the conductance tensor, the mode-I tension test of Sect. 4.1 is repeated. The mesh consists here of $200 \times 200$ quadratic $\mathrm{B}$-spline elements; the length-scale parameter arises 

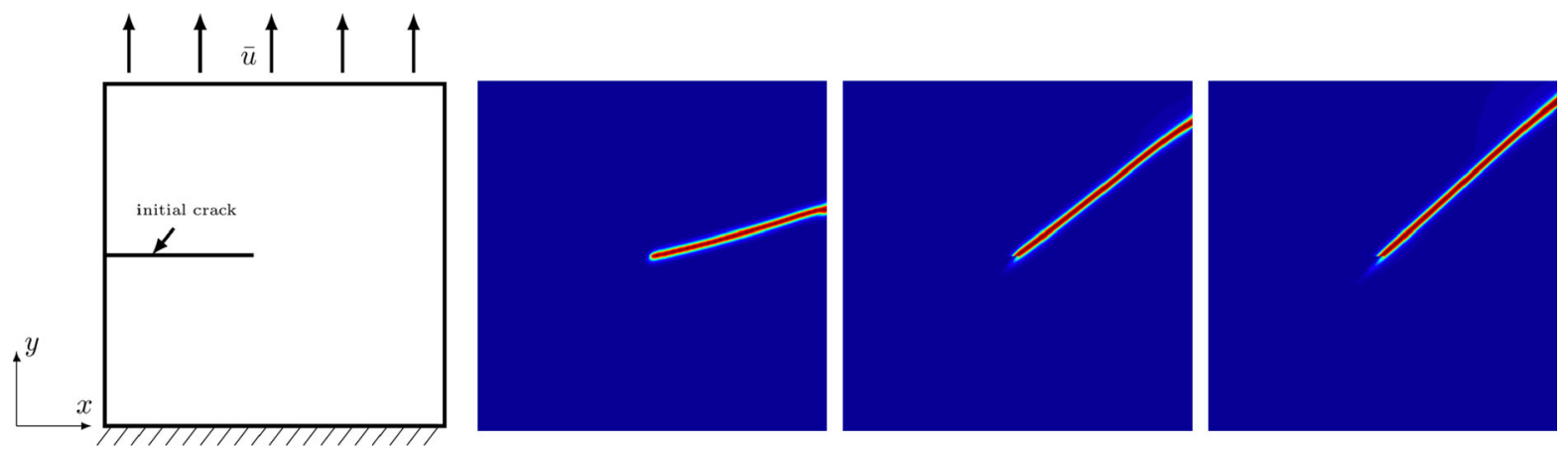

Fig. 9 Phase-field crack of the mode-I tension test for a material with direction vector $\boldsymbol{a}_{0}=(1 / \sqrt{2}, 1 / \sqrt{2})$ and weights $\alpha=1$, $\alpha=\sqrt{5}, \alpha=5$ (from left to right); crack driven by the $\lambda-\mu$ energy split

as $l_{c}=1 \mathrm{~mm}$. The preferred direction of the material is declined by an angle of $45^{\circ}$ which corresponds to a direction vector $\boldsymbol{a}_{0}=(1 / \sqrt{2}, 1 / \sqrt{2})$. Varying the parameter $\alpha$ with $\alpha \in\{1, \sqrt{5}, 5\}$ gives different weights to the inclination; the related crack paths are shown in Fig. 9. It is noticeable that with increasing $\alpha$ the crack deviates stronger in the preferred direction; for $\alpha \rightarrow \infty$ the crack would always go in direction of vector $\boldsymbol{a}_{0}$. This result is confirmed by other authors who presented similar approaches, cf. Teichtmeister et al. (2017) and Liu and Juhre (2018).

\subsubsection{Mode-II shear test with different weights and crack driving forces}

To take both tensile and shear components into account a mode-II shear test is simulated in the following. While the mesh and the material parameters remain the same, the boundary conditions on the upper side are adapted, prescribing a displacement in $x$-direction. The direction vector is given by $\boldsymbol{a}_{0}=(1,0)$ to simulate a preference in horizontal direction.

We perform the simulation with two different crack driving forces here, namely the energetic force with energy split (16) and the Rankine stress model (5). Comparing both crack paths we see a similar effect of the conductance tensor, i.e., the crack path is influenced and changes in the preferred direction. For bigger weights $\alpha$ the crack propagates stronger in the horizontal direction, see Figs. 10 and 11.

The main difference is that the crack computed with the $\lambda-\mu$ split is bent, likely because of the coupled influence of tension and shear, whereas the Rankine stress model results in a straight crack. The results coin- cide with the results of the tension test and confirm the effect of weight $\alpha$. The related load-deflection curves are presented in Fig. 12. In both cases it is observed that with growing weight the applied force and/or the prescribed displacement at cracking increase. We remark that this model has been used with the $\lambda-\mu$ energy split of linear elasticity in Clayton and Knap (2015), Teichtmeister et al. (2017) and Liu and Juhre (2018) for polycrystals but it works for deformations of rubbery material as well.

\subsubsection{Mode-I tension test with different directions}

In a next step we perform the mode-I tension test with different direction vectors. Based on the previous results the weight $\alpha$ is fixed at $5 \sqrt{2}, \alpha^{2}=50$. Two direction vectors are chosen, $\boldsymbol{a}_{01}=(1 / \sqrt{2}, 1 / \sqrt{2})$ and $\boldsymbol{a}_{02}=(1 / \sqrt{5},-2 / \sqrt{5})$. With both crack driving forces, energy split (16) and Rankine stress (5), we get coinciding results, exemplarily displayed in Fig. 13. The left plot shows the crack path for an isotropic material model, middle and right plot show the crack growing in the direction of $\boldsymbol{a}_{01}$ and $\boldsymbol{a}_{02}$. It can be observed that the cracks immediately grow in the preferred direction.

\subsection{Randomly weighted direction dependence}

Here we now combine both approaches to anisotropic crack growth, the stochastic field (32) and the conductance tensor (37). The crack resistance of the material is assumed to change locally, prescribed by a direction vector $\boldsymbol{a}=\alpha \boldsymbol{a}_{0}$. Because a random change in direction may easily result in a nearly isotropic behavior, 

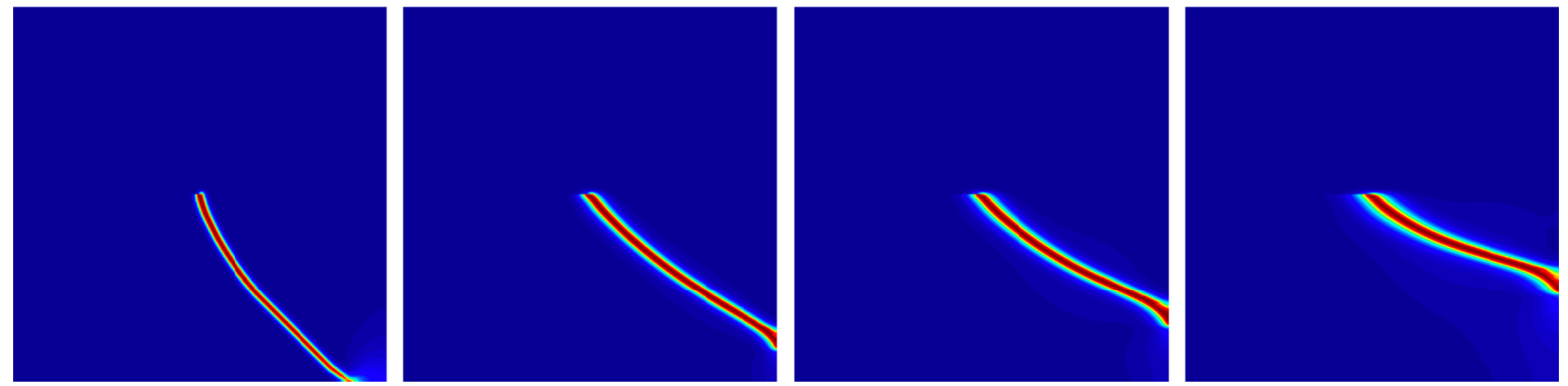

Fig. 10 Phase-field crack of the mode-II shear test for a material with direction vector $\boldsymbol{a}=(1,0)$ and different weights $\alpha=0, \alpha=\sqrt{5}$, $\alpha=\sqrt{10}$ and $\alpha=5$ (from left to right); crack driven by the $\lambda-\mu$ energy split
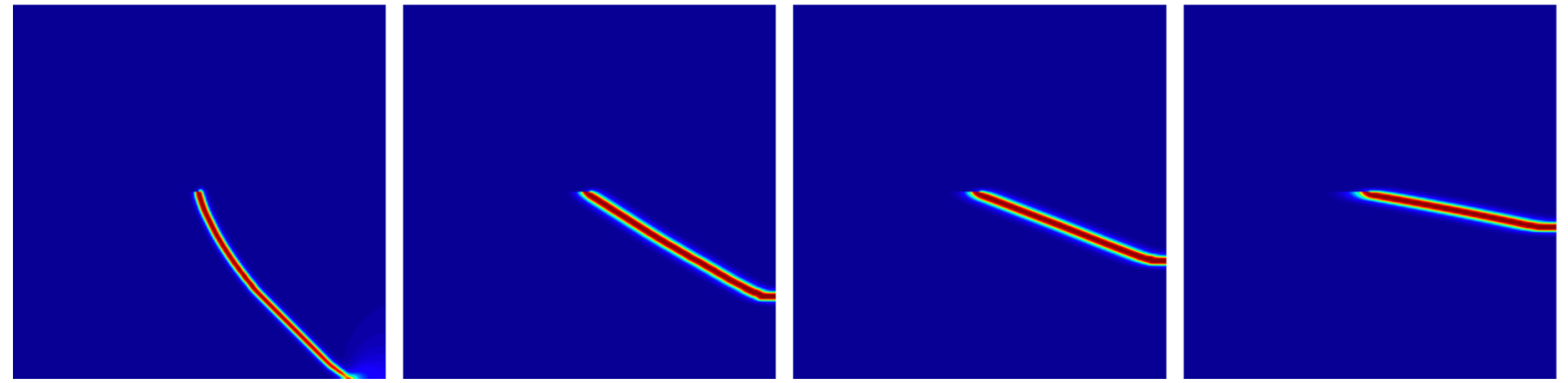

Fig. 11 Phase-field crack of the mode-II shear test for a material with direction vector $\boldsymbol{a}=(1,0)$ and different weights $\alpha=0, \alpha=\sqrt{5}$, $\alpha=\sqrt{10}$ and $\alpha=5$ (from left to right); crack driven by the Rankine stress
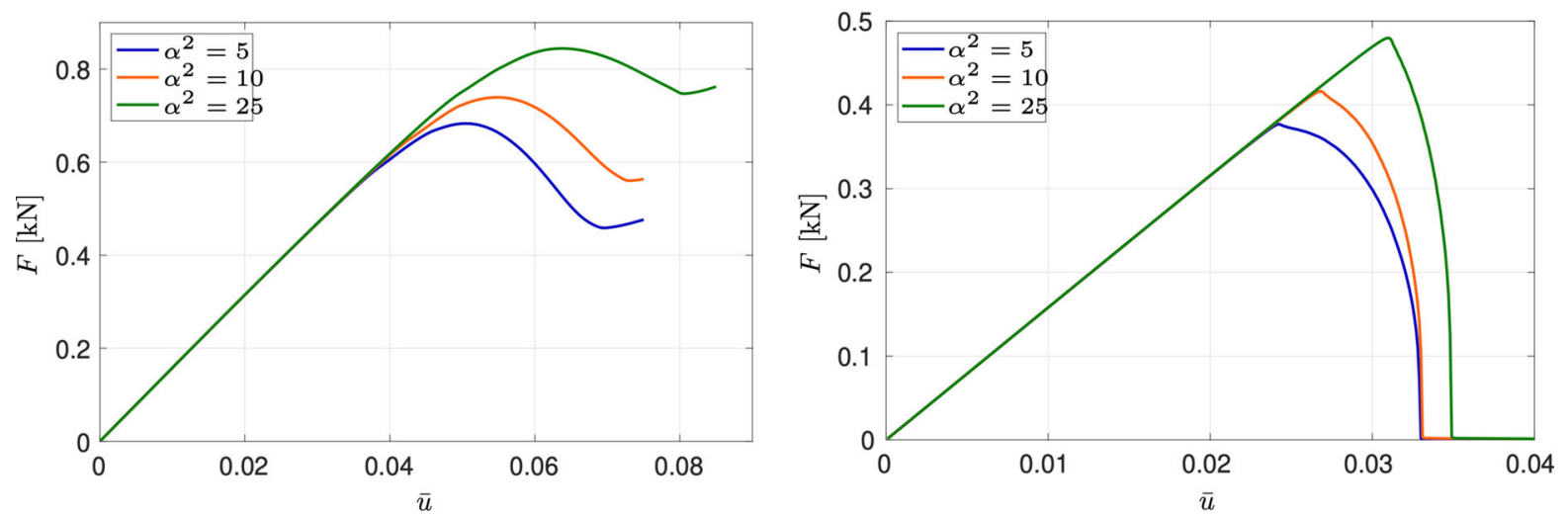

Fig. 12 Load-deflection curves of the mode-II shear test with the various weights $\alpha$

Fig. 13 Phase-field crack of the mode-I tension test for an isotropic material behavior and for an anisotropic crack resistance with different direction vectors

$\boldsymbol{a}_{01}=(1 / \sqrt{2}, 1 / \sqrt{2})$ and $\boldsymbol{a}_{02}=(1 / \sqrt{5},-2 / \sqrt{5})$ and length $\alpha=5 \sqrt{2}$ (from left to right); crack driven by the Rankine stress
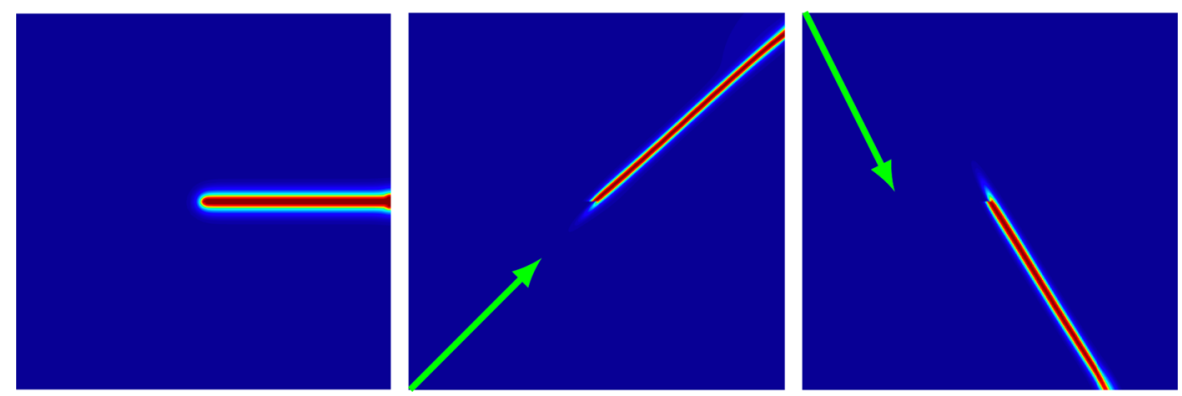
we define here a conductance direction $\boldsymbol{a}_{0}$ and change only its influence, namely the vector length. It shall be defined by the OSSRF, i.e $\alpha^{2}=\xi(\boldsymbol{x})$.

The effect is demonstrated by means of the modeII shear test of Sect. 4.2.2. The direction vector is $\boldsymbol{a}_{0}=(1,0)$ again, define a compliance in horizontal direction. The results are displayed in Fig 14. For the random field the eigenvectors and the Hurst indices are fixed but the amplitude of $\xi(\boldsymbol{x})$ varies between $[0,10]$ in the first case and between $[0,25]$ in the second case. It can be observed that the influence of anisotropy is differently strong, resulting in meaningful looking crack paths.

\section{Numerical Examples}

In this section we provide some sample calculations for pneumatic fracture in a material with layer-like anisotropic crack resistance. Such materials are, for example, shale formations, laminated safety glass, multiply rubber or vascular vessels. The crack growth is driven by the moving boundary model of Sect. 3.1 and includes now the direction dependent crack density function (37). In the following we will consider linear material behavior, but rather the theory can be also adapted to finite deformations.

\subsection{Pressure driven crack growth in plane stress}

We start with the example in Sect. 3.1 where a quadratic plate with a prescribed notch in its center is considered, see left plot of Fig. 15. The material parameters and boundary conditions remain unchanged. For crack anisotropy three conductance vectors are defined, all with relative length $\alpha=5 \sqrt{2}$. The directions are chosen as $\boldsymbol{a}_{01}=(1 / \sqrt{2}, 1 / \sqrt{2}), \boldsymbol{a}_{02}=(2 / \sqrt{5}, 1 / \sqrt{5})$ and $\boldsymbol{a}_{03}=(1 / \sqrt{5},-2 / \sqrt{5})$. The initial pressure is given by $p_{0}=10 \mathrm{~N} / \mathrm{mm}^{2}$.

The notch is initially horizontal and so the crack would expand as displayed in Fig. 2. Because of the preferred direction the crack here declines and propagates immediately in the preferred direction as shown in the three snapshots of Fig. 15. In the displayed anisotropic computations the crack driving force is based on the maximum of principal stress (5) and as soon as the critical stress is exceeded the crack grows in the preferred direction. The crack patterns for the energetic crack driving force are identical and therefore not displayed. In both cases the anisotropic crack resistance is modeled reasonably.

Next a layered structure is investigated, see Fig. 16. The mesh consists of $200 \times 200$ quadratic B-spline elements, all boundaries are fixed in each direction and the material parameters remain as previously introduced. The pressure is given by $p_{0}=10 \mathrm{~N} / \mathrm{mm}^{2}$ as well. In each layer another preferred direction occurs. The direction vectors are chosen alternatingly to be $\boldsymbol{a}_{01}=(1 / \sqrt{2}, 1 / \sqrt{2})$ and $\boldsymbol{a}_{02}=(-1 / \sqrt{2}, 1 / \sqrt{2})$. In the center of the plate a small notch is prescribed by fixing $z=1$. The propagating phase-field cracks are shown in Fig. 16 at different instants of time. It can be seen how the crack kinks at the layers' interfaces. The crack zigzags until it reaches the boundaries and so follows the prescribed anisotropic crack resistivity.

\subsection{Pressure driven crack growth in a cylinder with centered crack}

Although our examples have been plane, the proposed formulations are not restricted to two dimensions. In this subsection we examine the well known pennyshaped crack problem in three dimensions, studied for example in Lee et al. (2016), Miehe and Mauthe (2016) and Zhou et al. (2018). We consider a cylinder with a diameter of $50 \mathrm{~mm}$ and a height of $50 \mathrm{~mm}$. The mesh consists of 115000 linear brick elements (Lagrange ansatz functions). The material parameters remain as before, are based on linear material behavior and correspond to concrete, Table 1 . The outer boundaries of the structure are constrained in all directions. In the center of the specimen an initial crack is modeled by a squared surface where the phase-field parameter is set $z=1$. The mesh and the initial configuration are demonstrated in Fig. 17.

The pressure is induced by the moving boundary condition suggested of Sect. 3.1 with an amount of $p_{0}=10 \mathrm{~N} / \mathrm{mm}^{2}$ and an anisotropic crack resistance is assumed. In one example the direction vector is chosen to be $\boldsymbol{a}_{01}=(1 / \sqrt{3}, 1 / \sqrt{3}, 1 / \sqrt{3})$ so that the preferred direction is a spacial diagonal. In another example the direction vector is set to $\boldsymbol{a}_{02}=\frac{1}{\sqrt{27}}(1,5,1)$ which means that the $y$-direction is strongly preferred. The computed crack paths with the related isosurfaces are presented for $z=0.9$ in Fig. 18. We use the Rankine stress model (5) here, i.e., the crack surface grows as 

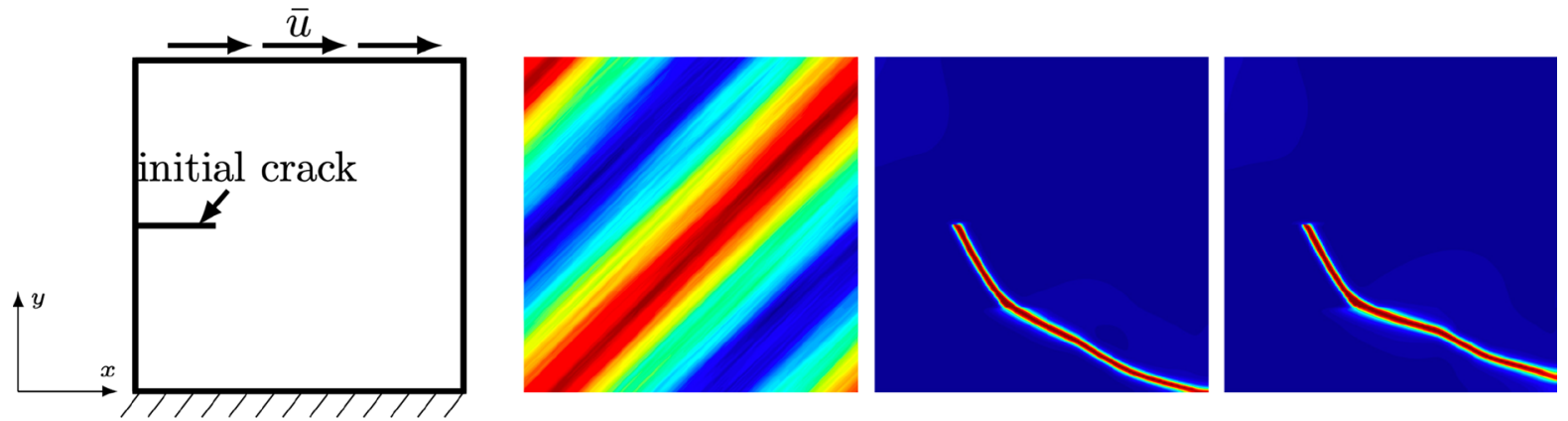

Fig. 14 Model of the adapted mode-II shear test; the applied random field $\xi(\boldsymbol{x})$; the phase-field crack path for $0 \leq \xi(\boldsymbol{x}) \leq 10$ and the phase-field crack path for $0 \leq \xi(\boldsymbol{x}) \leq 25$ (from left to right)
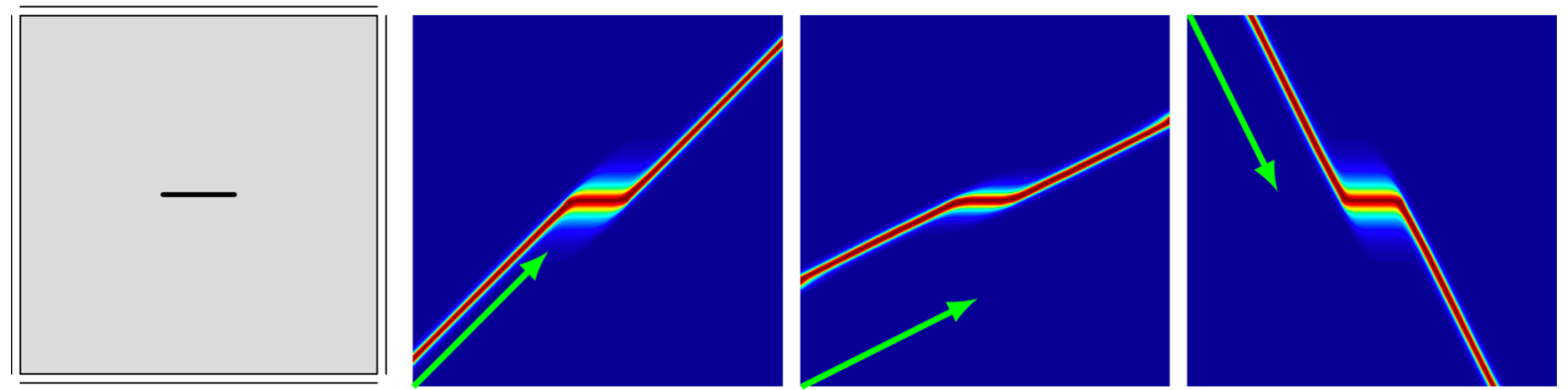

Fig. 15 Model (left) and pneumatic crack propagation paths for different direction vectors: $\boldsymbol{a}_{01}=(1 / \sqrt{2}, 1 / \sqrt{2}), \boldsymbol{a}_{02}=(2 / \sqrt{5}, 1 / \sqrt{5})$ and $\boldsymbol{a}_{03}=(1 / \sqrt{5},-2 / \sqrt{5})$
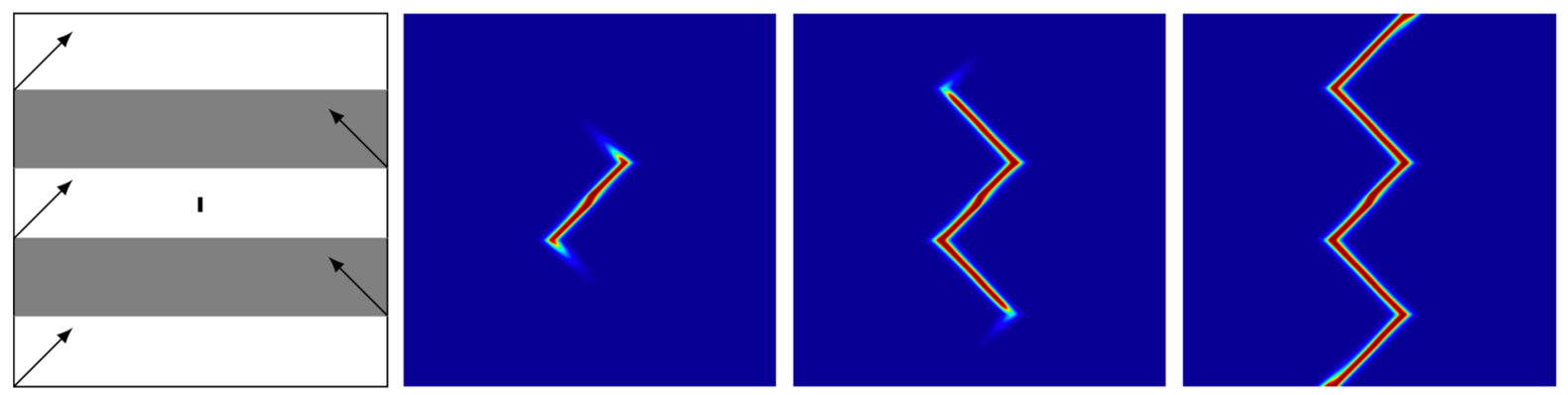

Fig. 16 Layered structure with different direction vectors: $\boldsymbol{a}_{01}=(1 / \sqrt{2}, 1 / \sqrt{2}), \boldsymbol{a}_{02}=(-1 / \sqrt{2}, 1 / \sqrt{2}), \alpha=5 \sqrt{2}$, and phase-field cracks at different time steps, which $t_{n} \in\{1,20,32\}$

soon as the critical stress is exceeded. It is observed that a crack surface arises which spreads through the whole cylinder and catches the anisotropic crack resistance.

\section{Concluding remarks}

In this contribution we present an extended phase-field model for pneumatic anisotropic fracture. The pressure driven crack growth is modeled with a moving bound- ary condition introduced in such a way that the pressure acts as a stress in the phase-field crack zone. The suitability of this approach is demonstrated with several examples. For modeling non-isotropic crack resistance two different approaches are discussed, namely a random field function in the Griffith energy and a structural conductance tensor in the crack density function. Both methods give the expected results but the conductance tensor approach is found to be more convenient for modeling layer-like materials. Eventually, regard- 
Fig. 17 Isosurface for $z=0.9$ (left) and the phase-field snapshot (right) in the initial configuration
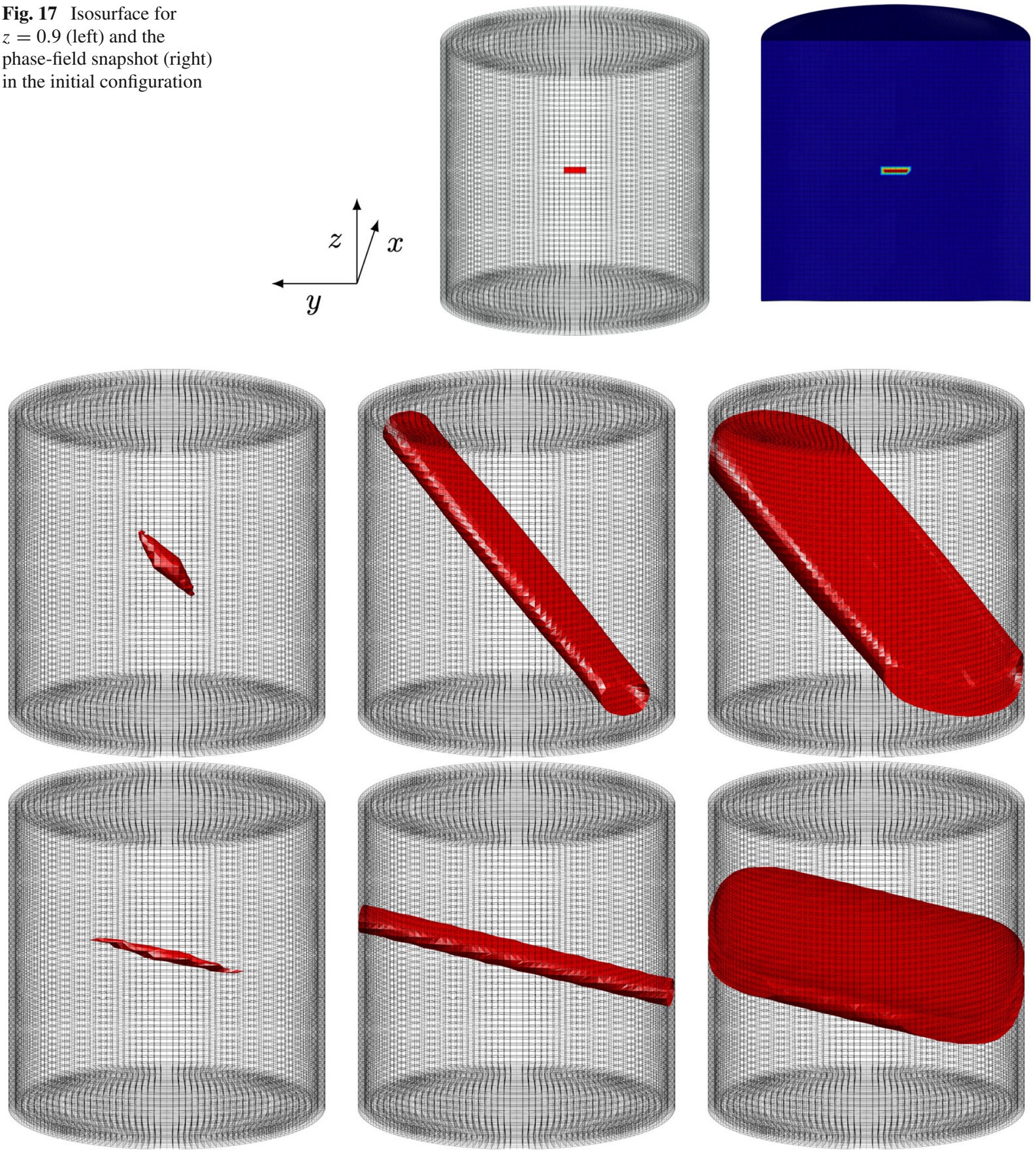

Fig. 18 Isosurface for $z=0.9$ for the pressure driven crack growth with a direction vector $\boldsymbol{a}_{\mathbf{0 1}}=\frac{1}{\sqrt{3}}(1,1,1)$ (upper row) and a direction vector $\boldsymbol{a}_{\mathbf{0 1}}=\frac{1}{\sqrt{27}}(1,5,1)$ (lower row) 
ing the applications in geological and biological materials both methods are combined.

To show the capabilities of the proposed strategy, two and three dimensional pneumatic fracture simulations have been performed with different phase-field crack driving forces. Hereby we found that all formulations can be used within our approach to pressuredriven crack growth leading to similar results and catching the direction dependent crack resistance properly.

Acknowledgements The authors gratefully acknowledge the support of the Deutsche Forschungsgemeinschaft under the project "Large-scale simulation of pneumatic and hydraulic fracture with a phase-field approach" (Grant DFG WE2525-4) as part of the Priority Program SPP 1748 (No. 255801726).

Funding Open Access funding enabled and organized by Projekt DEAL.

Open Access This article is licensed under a Creative Commons Attribution 4.0 International License, which permits use, sharing, adaptation, distribution and reproduction in any medium or format, as long as you give appropriate credit to the original author(s) and the source, provide a link to the Creative Commons licence, and indicate if changes were made. The images or other third party material in this article are included in the article's Creative Commons licence, unless indicated otherwise in a credit line to the material. If material is not included in the article's Creative Commons licence and your intended use is not permitted by statutory regulation or exceeds the permitted use, you will need to obtain permission directly from the copyright holder. To view a copy of this licence, visit http://creativecommons.org/licenses/ by $/ 4.0 /$.

\section{References}

Abdollahi A, Arias I (2012) Phase-field modeling of crack propagation in piezoelectric and ferroelectric materials with different electromechanical crack conditions. J Mech Phys Solids 60(12):2100-2126

Adda-Bedia M, Arias R, Amar MB, Lund F (1999) Generalized Griffith criterion for dynamic fracture and the stability of crack motion at high velocities. Phys Rev E 60(2):2366

AlTammar MJ, Sharma MM, Manchanda R (2018) The effect of pore pressure on hydraulic fracture growth: an experimental study. Rock Mech Rock Eng 51(9):2709-2732

Ambati M, De Lorenzis L (2016) Phase-field modeling of brittle and ductile fracture in shells with isogeometric NURBSbased solid-shell elements. Comput Methods Appl Mech Eng 312:351-373

Ambrosio L, Tortorelli VM (1990) Approximation of functionals depending on jumps by elliptic functionals via $\Gamma$ convergence. Commun Pure Appl Math 43(8):999-1036. https://doi.org/10.1002/cpa.3160430805

Amor H, Marigo JJ, Maurini C (2009) Regularized formulation of the variational brittle fracture with unilateral contact: numerical experiments. J Mech Phys Solids 57(8):12091229

Anders D, Hoffmann A, Scheffler HP, Weinberg K (2011) Application of operator-scaling anisotropic random fields to binary mixtures. Phil Mag 91(29):3766-3792

Bilgen C, Weinberg K (2019) On the crack-driving force of phase-field models in linearized and finite elasticity. Comput Methods Appl Mech Eng 353:348-372

Bilgen C, Hennig P, Kästner M, Weinberg K (2018) Numerical simulation of crack propagation in an anisotropic medium. Proc Appl Math Mech 18(1):e201800308

Bleyer J, Alessi R (2018) Phase-field modeling of anisotropic brittle fracture including several damage mechanisms. Comput Methods Appl Mech Eng 336:213-236

Borden MJ, Verhoosel CV, Scott MA, Hughes TJR, Landis CM (2012) A phase-field description of dynamic brittle fracture. Comput Methods Appl Mech Eng 217-220:77-95

Bourdin B, Francfort GA, Marigo JJ (2000) Numerical experiments in revisited brittle fracture. J Mech Phys Solids 48(4):797-826

Bourdin B, Francfort GA, Marigo JJ (2008) The variational approach to fracture. J Elast 91(1-3):5-148

Bourdin B, Chukwudozie CP, Yoshioka K (2012) A variational approach to the numerical simulation of hydraulic fracturing. In: SPE annual technical conference and exhibition. Society of petroleum engineers

Clayton JD, Knap J (2015) Phase field modeling of directional fracture in anisotropic polycrystals. Comput Mater Sci 98:158-169

Ghamgosar M, Williams DJ, Erarslan N (2015) Effect of anisotropy on fracture toughness and fracturing of rocks. In: 49th US rock mechanics/geomechanics symposium. American Rock Mechanics Association

Ghilardi G, Scorza R, Bortolani E, De Monti M, Longhi F, Ruberti U et al (1993) Rupture of abdominal aortic aneurysms into the major abdominal veins. J Cardiovasc Surg 34:39

Hansen-Dörr AC, Hennig P, Weinberg K, Kästner M (2017) A phase field model for interface failure. Proc Appl Math Mech 17(1):249-250

Heider Y, Markert B (2017) A phase-field modeling approach of hydraulic fracture in saturated porous media. Mech Res Commun 80:38-46

Henry H, Levine H (2004) Dynamic instabilities of fracture under biaxial strain using a phase field model. Phys Rev Lett 93:105505

Hesch C, Gil AJ, Ortigosa R, Dittmann M, Bilgen C, Betsch P, Franke M, Janz A, Weinberg K (2017) A framework for polyconvex large strain phase-field methods to fracture. Comput Methods Appl Mech Eng 317:649-683

Holzapfel GA (2006) Nonlinear solid mechanics-a continuum approach for engineering. Wiley, New York

https://www.healthtestingcenters.com/ what-you-need-know-about-cholesterol-and-cardiac-disease/. Accessed 24 Oct 2020

Karma A, Kessler DA, Levine H (2001) Phase-field model of mode III dynamic fracture. Phys Rev Lett 81:045501

Kuhn C, Müller R (2010) A continuum phase field model for fracture. Eng Fract Mech 77:3625-3634

Kuhn C, Noll T, Müller R (2016) On phase field modeling of ductile fracture. Surv Appl Math Mech 39(1):35-54 
Lee S, Wheeler MF, Wick T (2016) Pressure and fluid-driven fracture propagation in porous media using an adaptive finite element phase field model. Comput Methods Appl Mech Eng 305:111-132

Li B, Maurini C (2019) Crack kinking in a variational phase-field model of brittle fracture with strongly anisotropic surface energy. J Mech Phys Solids 125:502-522

Li B, Peco C, Millán D, Arias I, Arroyo M (2015) Phase-field modeling and simulation of fracture in brittle materials with strongly anisotropic surface energy. Int J Numer Meth Eng 102(3-4):711-727

Liu Z, Juhre D (2018) Phase-field modelling of crack propagation in anisotropic polycrystalline materials. Procedia Struct Integr 13:787-792

Luo C, Ehlers W (2015) Hydraulic fracturing based on the theory of porous media. Proc Appl Math Mech 15(1):401-402

Marsden JE, Hughes TJR (1994) Mathematical foundations of elasticity. Courier Corporation

Miehe C, Mauthe S (2016) Phase field modeling of fracture in multi-physics problems. Part III. Crack driving forces in hydro-poro-elasticity and hydraulic fracturing of fluidsaturated porous media. Comput Methods Appl Mech Eng 304:619-655

Miehe C, Hofacker M, Welschinger F (2010) A phase field model for rate-independent crack propagation: robust algorithmic implementation based on operator splits. Comput Methods Appl Mech Eng 199:2765-2778

Miehe C, Mauthe S, Teichtmeister S (2015a) Minimization principles for the coupled problem of Darcy-Biot-type fluid transport in porous media linked to phase field modeling of fracture. J Mech Phys Solids 82:186-217

Miehe C, Schänzel LM, Ulmer H (2015b) Phase-field modeling of fracture in multi-physics problems. Part I. Balance of crack surface and failure criteria for brittle crack propagation in thermo-elasitc solids. Comput Methods Appl Mech Eng 294:449-485

Mikelic A, Wheeler MF, Wick T (2015a) A phase-field method for propagating fluid-filled fractures coupled to a surrounding porous medium. Multisc Model Simul 13(1):367-398

Mikelić A, Wheeler MF, Wick T (2015b) Phase-field modeling of a fluid-driven fracture in a poroelastic medium. Comput Geosci 19(6):1171-1195

Mikelić A, Wheeler MF, Wick T (2019) Phase-field modeling through iterative splitting of hydraulic fractures in a poroelastic medium. GEM-Int J Geomath 10(1):2
Sargado JM, Keilegavlen E, Berre I, Nordbotten JM (2018) Highaccuracy phase-field models for brittle fracture based on a new family of degradation functions. J Mech Phys Solids 111:458-489

Sneddon LN, Lowengrub M (1969) SIAM—crack problems in the classical theory of elasticity. Wiley, New York

Sneddon LN, Mott NF (1946) The distribution of stress in the neighbourhood of a crack in an elastic solid. Proc R Soc Lond Ser A 187(1009):229-260

Teichtmeister S, Kienle D, Aldakheel F, Keip MA (2017) Phase field modeling of fracture in anisotropic brittle solids. Int $\mathbf{J}$ Non-Linear Mech 97:1-21

Thomas M, Bilgen C, Weinberg K (2020) Analysis and simulations for a phase-field fracture model at finite strains based on modified invariants. J Appl Math Mech. https://doi.org/ 10.1002/zamm.201900288

Verhoosel CV, de Borst R (2013) A phase-field model for cohesive fracture. Int $\mathrm{J}$ Numer Methods Eng

Weinberg K, Hesch C (2015) A high-order finite-deformation phase-field approach to fracture. Contin Mech Thermodyn. https://doi.org/10.1007/s00161-015-0440-7

Wheeler M, Wick T, Wollner W (2014) An augmentedLagrangian method for the phase-field approach for pressurized fractures. Comput Methods Appl Mech Eng 271:6985. https://doi.org/10.1016/j.cma.2013.12.005

Wilson ZA, Landis CM (2016) Phase-field modeling of hydraulic fracture. J Mech Phys Solids 96:264-290

Yamashima T, Friede R (1984) Why do bridging veins rupture into the virtual subdural space? J Neurol Neurosurg Psychiatry $47(2): 121-127$

Zhou S, Zhuang X, Rabczuk T (2018) A phase-field modeling approach of fracture propagation in poroelastic media. Eng Geol 240:189-203

Publisher's Note Springer Nature remains neutral with regard to jurisdictional claims in published maps and institutional affiliations. 